\title{
Research on Intelligence Express Logistics System Based on RFID
}

\author{
Yuan Feng \\ School of Computer and Communication Engineering \\ Zhengzhou University of Light Industry \\ Zhengzhou, China \\ e-mail: fy@zzuli.edu.cn
}

\section{Zengyu Cai}

School of Computer and Communication Engineering Zhengzhou University of Light Industry

Zhengzhou, China

e-mail: czy@zzuli.edu.cn

\author{
Fenglong $\mathrm{Wu}$ \\ Department of Software \\ Zhengzhou University of Light Industry \\ Zhengzhou, China \\ e-mail: wufenglong@zzuli.edu.cn
}

\section{Baowei Zhang}

School of Computer and Communication Engineering Zhengzhou University of Light Industry

Zhengzhou, China

e-mail: bwzh@zzuli.edu.cn

\begin{abstract}
By combining information security, computer network technology, wireless communication technology and genetic algorithm, this paper puts forward a design scheme of intelligence express logistics system based on RFID and conducts a study on its overall framework and system flow, which emphatically discusses key technologies such as intelligence path planning, the safety of on-vehicle data and electronic distribution invoice and the management and restriction of user privilege. The application of this system indicates that it has perfect function, high intelligence and security.
\end{abstract}

Keywords- express logistics; intelligence logistics; RFID; safety guarantee

\section{INTRODUCTION}

Express logistics technology has entered the stage of intelligence logistics research, and application and electronic logistics has become an indispensable part of e-commerce[1]. RFID (Radio Frequency Identification) has such advantages as long reading distance, strong privacy, large memory quantity, high anti-interfering and long life [2-4]. Among them the features of long reading distance and large memory quantity make its application in logistics field more and more popular and its influence on Intelligence Logistics more important [5]. This paper presents a design scheme of logistics management system which combines computer network technology, wireless communication technology, and genetic algorithm with RFID technology, introduces its system architecture and work flow in detail, makes a further study on it and then explores the related key technologies.

\section{GENERAL STRUCTURE OF INTELLIGENCE EXPRESS LOGISTICS SYSTEM BASED ON RFID}

The basic work flow of express logistics mainly consists of warehousing, distributing, ex-warehousing, transporting and delivering. The general scheme of intelligence logistics system based on RFID is shown in Fig.1. Logistics management system based on RFID mainly includes three parts: the overall network, the branch or warehouse network and the distribution and mobile terminal. As shown in Fig.1, the overall network can connect with the branch or warehouse network via public network, and the distribution and mobile terminal can either connect with the warehouse management terminal through wireless communication agreement, or communicate with the local server by GPRS. The system is mainly composed of centre server, local server in warehouse, management machine, warehouse management terminal, distribution and mobile terminal and standard RFID pallet.

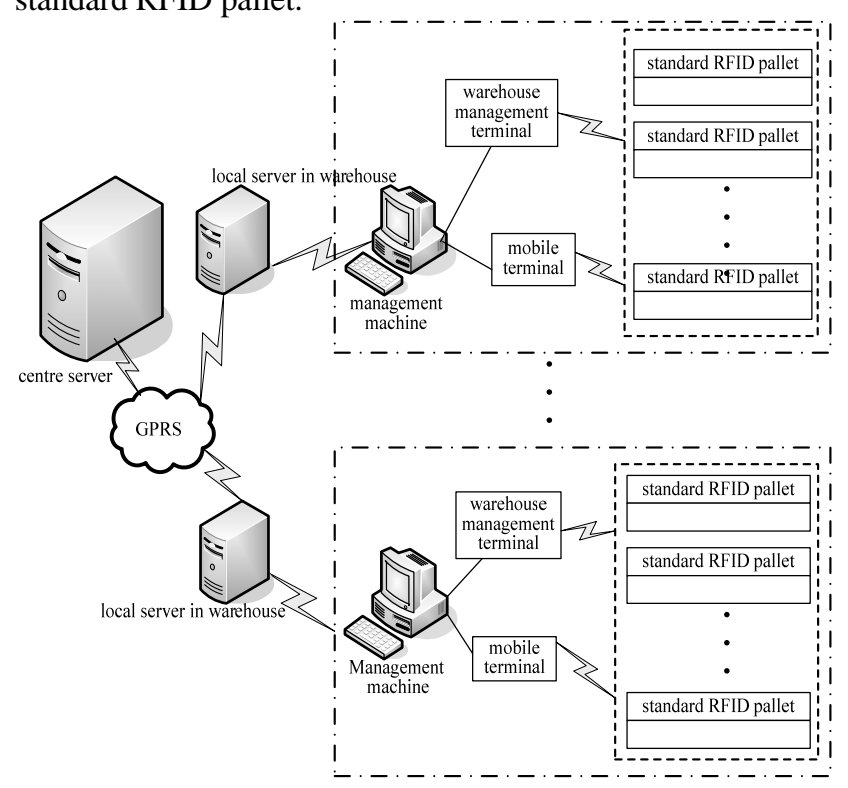

Figure 1. The General Structure of Intelligence Express Logistics System based on RFID 


\section{SYSTEM FLOW OF EXPRESS LOGISTICS BASED ON RFID}

The basic work flow of express logistics management system based on RFID technology mainly consists of accepting order, warehousing, sorting, pallet distributing, selling out, distributing and delivery, each of them store or update the related information through logistics management system which is indispensable in the whole process. As is shown in Fig.2, the flow begins with the order of the customer and ends up with the delivery.

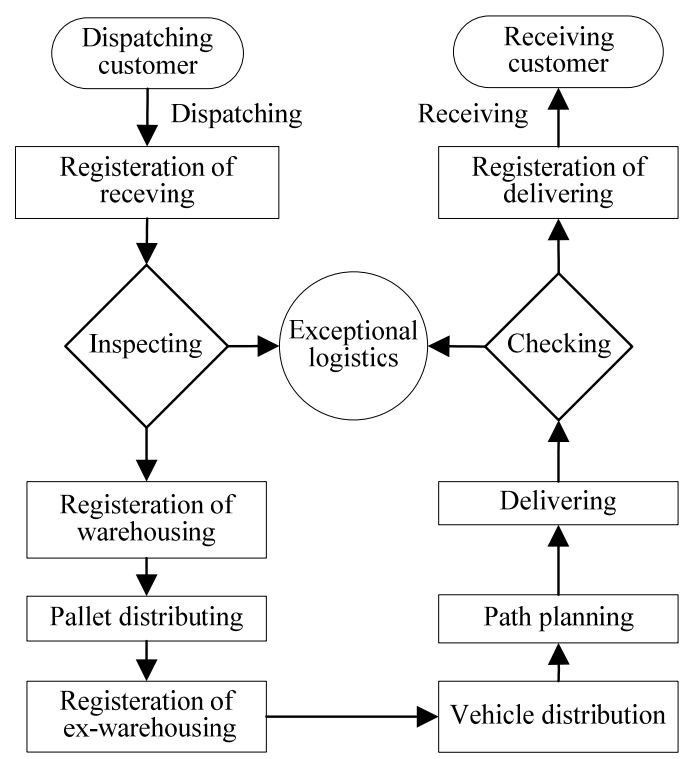

Figure 2. Basal process of Intelligence Express Logistics System based on RFID

The basic work flow of logistics management system based on RFID technology mainly consists of dispatching, receiving, warehousing, sorting, pallet distributing, selling out, distributing and delivery, each of them store or update the related information through logistics management system which is indispensable in the whole process. As is shown in Fig.2, the flow begins with the order of the customer and ends up with the delivery.

1) Dispatching.

First, the customer submits the order and requests for dispatching.

2) Registration of receiving.

The receiver accepts the goods and inspects them to judge if they can be mailed by express. If they can, they will be admitted into the registration; if not, they will be included into exceptional logistics.

3) Registration of warehousing.

After the goods being received by the receiver, the RFID reader at the entrance of express distribution centre can collect the information of all goods and store them in the data base of data server machine automatically, and then the registration is finished.

4) Pallet distributing.
In this process, first, the goods will be sorted according to user's order; then they will be distributed to the pallets according to the capacity of each pallet, the amount of the goods already loaded and the destination of the goods, the related information will also be recorded and the location and state of the goods be updated in data base.

5) Registration of ex-warehousing.

When the pallets are distributed, the ex-housing will be registered at the exit of the distribution centre. The information of the goods will be collected by the RFID reader automatically and recorded in data base.

6) Vehicle distribution.

The vehicle of each pallet should be determined according to the information of the goods. For example, the rule to convey the pallets which have close destination in a same vehicle.

7) Path planning.

The path of the vehicle should be determined by using intellectual technology in accordance with the information of the goods.

8) Delivering.

When the vehicle arrives at a distribution centre, the pallet should be identified by on-vehicle RFID reader and then be given to the customer, in order to ensure the goods are in keeping with the order completely. The goods will enter exceptional logistics management process if they have any problem. If they can, they will be admitted into the registrations; if not, they will be included into exceptional logistics.

9) Registrations of delivering.

It records the delivery information by on-vehicle RFID reader to communicate with logistics management system.

10) Receiving.

The receiving customer accepts the goods.

The exceptional logistics management refers to the emergence of the events which are not meet the requirements or incorrect and the interruption of the logistics flow which means that the goods will back to previous link or be returned.

\section{ANALySES ON Key TECHNOLOGIES}

\section{A. Intelligence Path Planning based on Genetic Algorithm}

In the respect of intelligence, intelligence pallet distributing and intelligence path planning are mainly involved. In intelligence pallet distributing, there are many factors need to be taken into consideration, such as the quantity, capacity and destination of the goods, the size and capacity of the pallet, the distance between destinations and the co-existence of the goods. Due to the relatively maturity of mathematical programming method and the limitation of goods' quantity in one warehouse, it is a viable method to solve this problem; Intelligence path planning involves the problems of the quantity of the goods, destination, electronic map and time limitation. The traditional solution is to use mathematical programming method of which the speed will drop dramatically when the scale of the system is enormous. Some problems even can only be solved theoretically instead 
of practically. Thus, the genetic algorithm is used to make path planning optimized.

This paper adopts natural number coding method, initial chromosome producing stochastic method, fitness function objective function reciprocal method, population choosing roulette selection operator; the default crossover rate is 0.7 , mutation operation is realized by exchanging two non-zero genes' positions in one same chromosome randomly with a mutation probability of 0.001 . The genetic algorithm chromosome population of which average cost decreases gradually with the increasing of hereditary algebra and convergence rate has good function in logistics vehicle dispatching field has great application prospect.

\section{B. Realization of System security}

Due to the complexity of the environment of this module and the feature of public network data transmissions, safety precautions must be taken to ensure data security.

1) Security of on-vehicle data.

The data must be enciphered in storage and restricted in system usage, in order to prevent the data loss or leak. This system uses triple DES (Data Encryption Standard, DES) which needs password in landing.

2) Security of electronic distribution invoice.

SSL (Security Socket Layer, SSL) safety agreement needs to be used in this process to ensure safety.

3) User privilege management and restriction.

In this system, receiver, distributor, local system manager, high-level system manager are involved, all levels of users must be managed strictly to prevent excess of authority. The integral security of the system and the privacy of the information need to be protected by complete log system which stores various of logs rigorously and can only be deleted by system manager after a certain time.

\section{CONCLUSION}

In this paper, intelligence logistics system which combines RFID, information security, computer network technology and artificial intelligence achieves the whole course logistics management. An experiment has been done in an express company after which the users agree unanimously that this system has the following three features.

1) Perfect function

This system can manage all the links including dispatching, receiving, warehousing, sorting, pallet distributing, ex-warehousing, and distributing, delivery and return and it achieves whole course management.

2) High intelligence

This system can read the related information of RFID pallet conveniently, pick up the optimum path rapidly by genetic algorithm, and improve the quality of logistics distribution and save transportation cost.

3) High safety

Triple DES which enciphers the on-vehicle data ensures the data safety: SSL agreement of secure transmission is used in all internet data transmission.

\section{REFERENCES}

[1] K. V. RAO, P. V. NIKITIN, and S. F. LAM, "Antenna design for UHF RFID tags: a review and a practical application,” IEEE Transaction on Antenna Propagation, vol. 53, Dec. 2005, pp.38703876.

[2] E.W.T. Ngai, D.C.K. Chau, J.K.L. Poon, A.Y.M. Chan, B.C.M. Chan, and W.W.S. Wu, "Implementing an RFID-based manufacturing process management system: Lessons learned and success factors,” Journal of Engineering and Technology Management, Vol. 29, Jan.Mar. 2012, pp.112-130.

[3] Qing Hua Zhang, and et al., "Research on Home Appliance Lifecycle Information Management System Based on RFID," Applied Mechanics and Materials, 2012, pp.157-158, 254-257.

[4] Weixing Su, Kunyuan Hu, Lei Zhang, and Lianbo Ma, “A RFIDBased Material Supply Management System in Automatic Vehicle Assembly Streamline," Information Technology and Computer Science (ITCS 2009), IEEE Press, Jul. 2009, pp. 259 - 262.

[5] Tan Kah Ling, C.K.M. Lee, and William Ho, "The analysis and case studies of successful express logistics companies,” International Journal of Value Chain Management, Vol. 3, Jan. 2009, pp. 20-35. 